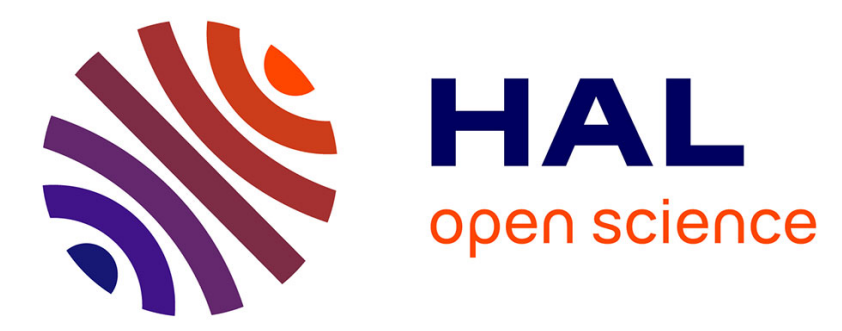

\title{
Optical frequency comb cavity ring-down spectroscopy using a time-resolved Fourier transform spectrometer
}

Romain Dubroeucq, Aleksander Gluszek, Grzegorz Soboń, Lucile Rutkowski

\section{To cite this version:}

Romain Dubroeucq, Aleksander Gluszek, Grzegorz Soboń, Lucile Rutkowski. Optical frequency comb cavity ring-down spectroscopy using a time-resolved Fourier transform spectrometer. Optical Sensors and Sensing Congress, Jul 2021, online, United States. hal-03332436

\section{HAL Id: hal-03332436 \\ https://hal.science/hal-03332436}

Submitted on 2 Sep 2021

HAL is a multi-disciplinary open access archive for the deposit and dissemination of scientific research documents, whether they are published or not. The documents may come from teaching and research institutions in France or abroad, or from public or private research centers.
L'archive ouverte pluridisciplinaire HAL, est destinée au dépôt et à la diffusion de documents scientifiques de niveau recherche, publiés ou non, émanant des établissements d'enseignement et de recherche français ou étrangers, des laboratoires publics ou privés. 


\title{
Optical frequency comb cavity ring-down spectroscopy using a time-resolved Fourier transform spectrometer Romain Dubroeucq ${ }^{1}$, Aleksander Gluszek ${ }^{2}$, Grzegorz Soboń ${ }^{2}$, Lucile Rutkowski ${ }^{1 *}$ \\ 1. Univ Rennes, CNRS, IPR (Institut de Physique de Rennes) - UMR 6251, F-35000 Rennes, France \\ 2. Laser \& Fiber Electronics Group, Faculty of Electronics, Wroctaw University of Science and Technology, 50-370 Wroclaw, Poland \\ *lucile.rutkowski@univ-rennes1.fr
}

\begin{abstract}
We show the preliminary results of cavity ring-down spectroscopy based on a nearinfrared frequency comb source and retrieve the multiplexed decays using a time-resolved fastscanning Fourier transform spectrometer. (C) 2021 The Author(s)
\end{abstract}

\section{Introduction}

Cavity ring-down spectroscopy (CRDS) is one of the most sensitive technique to measure absorption. Nowadays, it is most often based on continuous wave sources, which offer a high spectral density and a high resolution but lead to long measurement times due to their tuning delays. Instead, a CRDS setup based on a broadband light source would offer the high sensitivity together with the advantages of a multiplex acquisition. Previous works have explored the possibility of analysing the broadband cavity decay using time-resolved FTIR and a pulsed dye laser [1], and using a virtual image phased array combined with a frequency comb source [2], but none of them succeeded in achieving a sensitivity high enough to compete with the other broadband spectrometers based on optical cavities. Here, we demonstrate broadband CRDS based on an optical frequency comb source and detected using a time-resolved Fourier transform spectrometer FTS. We exploit the ability to lock the comb to the ring-down cavity to ensure a high throughput for the FTS, allowing a fast acquisition of all the cavity decays simultaneously.

\section{Experimental setup}

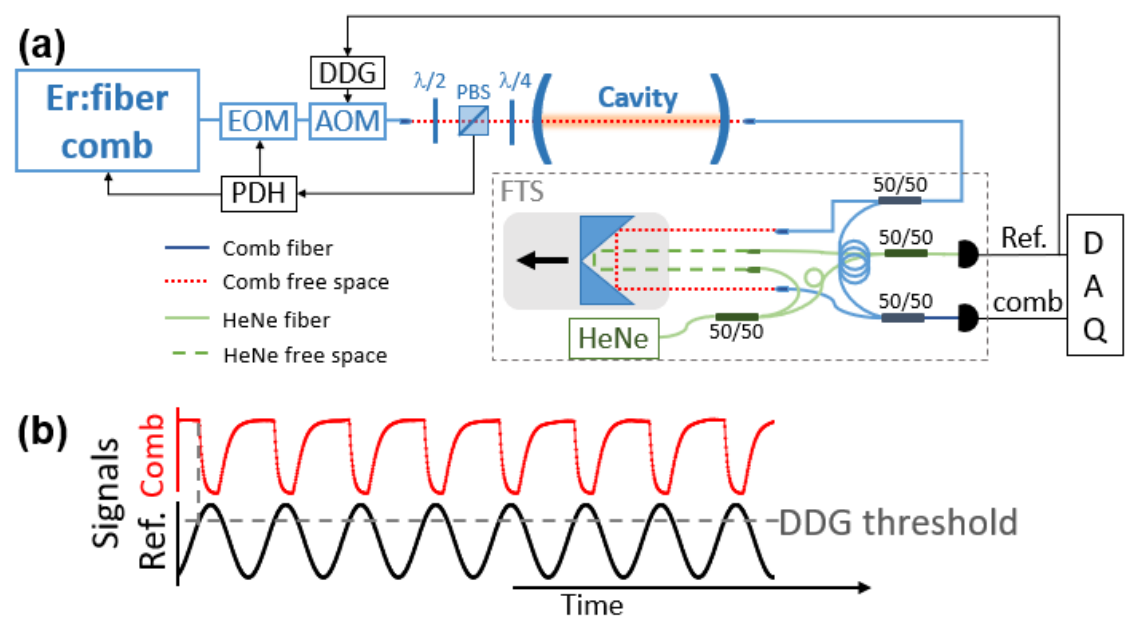

Fig. 1. (a) Experimental setup: EOM, electro-optic modulator; AOM, acousto-optic modulator; $\lambda / 2$, half waveplate; PBS, polarizing beam splitter; $\lambda / 4$, quarter waveplate; PDH, Pound-Drever-Hall locking electronics; DDG, digital delay generator; DAQ, acquisition card. (b) Schematic of the acquisition.

The experimental setup is depicted in Fig. 1(a). The frequency comb is a Erbium-fiber source with a repetition rate of $125 \mathrm{MHz}$, covering $30 \mathrm{~nm}$ around $1560 \mathrm{~nm}$ [3]. The ring-down cavity has a finesse of $\sim 450$, and a length of 1.2 $\mathrm{m}$. The comb repetition rate is locked to the cavity free spectral range using the Pound-Drever-Hall technique while the offset frequency is free-running. The cavity transmission is analyzed using a fast-scanning FTS. The FTS relies on fibered couplers and splitters instead of the conventional free-space beam splitter, allowing for a more compact setup. The comb beam is first splitted into two arms of equal power. One of the arms is collimated in free space before reflecting on a corner-cube mounted on a linear translation stage. The reflected beam is coupled back in fiber and is combined with the second arm to yield the interferogram. The optical path difference of the interferometer is 
calibrated using a frequency-stabilized He-Ne laser which propagates on a similar path and both the HeNe reference and the comb interferograms are recorded simultaneously.

The CRDS events are synchronised to the FTS scan by sending the reference signal from the He-Ne laser to a digital delay generator which triggers an acousto-optic modulator and switches off the comb light. The bandwidth of the locking electronics has been adjusted so that it is not affected by the chopping of the comb light. A subset of the raw data acquired is shown in Fig. 1(b). The comb signal contains the successive decays measured with a $25 \mathrm{MHz}-$ bandwidth photodetector at optical path differences separated by a constant step of $633 \mathrm{~nm}$.

\section{Preliminary results}

The 3-dimensional interferogram shown in Fig. 2(a) is retrieved from the raw data in post-processing. The raw data is truncated in pieces corresponding to one period of the HeNe interferogram and reassembled in a matrix where each intensity point correspond to a delay after the AOM trigger and an optical path difference. Figure 2(a) shows the center part of the burst with the dc component of the interferogram subtracted at each delay. Taking the fast Fourier transform of the interferograms obtained at given delays after the trigger yields the spectrum shown in Fig. 2(b), featuring the cavity decay at each spectral element. The total optical path difference acquired is $21 \mathrm{~cm} \mathrm{long}$, yielding a spectral resolution of $1.4 \mathrm{GHz}$. The ring-down obtained at $6405 \mathrm{~cm}^{-1}$ is plotted in Fig. 2(c) together with the fitted exponential decay model. The residuum of the fit has a standard deviation of $1.5 \times 10^{-3}$, thus the SNR of the decay reaches 600. The ring-down time retrieved from this fit is $0.497 \mu \mathrm{s}$, in good agreement with the mirror reflectivity of $99.3 \%$ specified by the manufacturer (Layertec, coating 114951).
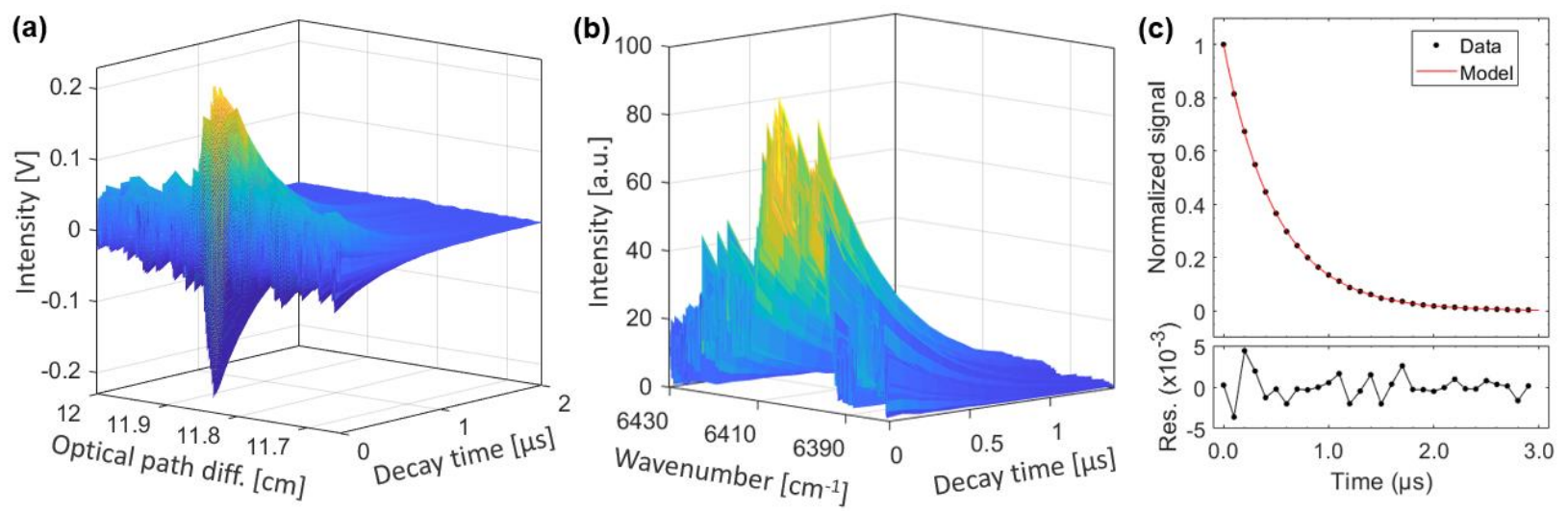

Fig. 2. (a) 3D interferogram obtained from the data, cut with the adapted optical path difference step and reassembled. (b) 3D spectrum obtained when taking the FFT of the interferogram for each decay time, showing the spectral distribution of the decays. (c) Measured ring-down at $6405 \mathrm{~cm}^{-1}$ (markers) together with the fitted exponential decay model (red line) and residuum (bottom panel).

\section{Conclusion and outlook}

We have demonstrated cavity ring-down spectroscopy based on an optical frequency comb source and a timeresolved FTS. This technique will be implemented using a cavity with a higher finesse and a more powerful laser source, opening up for high-sensitivity spectroscopy. The technique is also fully compatible with the sub-nominal Fourier transform spectroscopy method [4], which will allow high frequency precision and comb mode resolution.

Acknowledgements: This work is supported by the ANR project CECoSA (ANR-19-CE30-0038), the National Physics Institute (CNRS), and Rennes Metropole. G.S. and A.G. are supported by the Foundation for Polish Science (First TEAM/2017-4/39).

\section{References}

[1] R. Engeln and G. Meijer, “A Fourier transform cavity ring down spectrometer”, Rev. Sci. Instr. 67, 2708 (1996).

[2] M. J. Thorpe, K. D. Moll, R. J. Jones, B. Safdi, J. Ye, "Broadband cavity ringdown spectroscopy for sensitive and rapid molecular detection", Science 311(5767), 1595-1599 (2006).

[3] A. Głuszek, F. Senna Vieira, A. Hudzikowski, A. Wąż, J. Sotor, A. Foltynowicz, G. Soboń, "Compact mode-locked Er-doped fiber laser for broadband cavity-enhanced spectroscopy," Appl. Phys. B 126, 137 (2020).

[4] L. Rutkowski, P. Maslowski, A.C. Johansson, A. Khodabakhsh and A. Foltynowicz, "Optical frequency comb Fourier transform spectroscopy with sub-nominal resolution and precision beyond the Voigt profile", J. Quant. Spectrosc. Radiat. Transfer, 204, 63 (2018). 